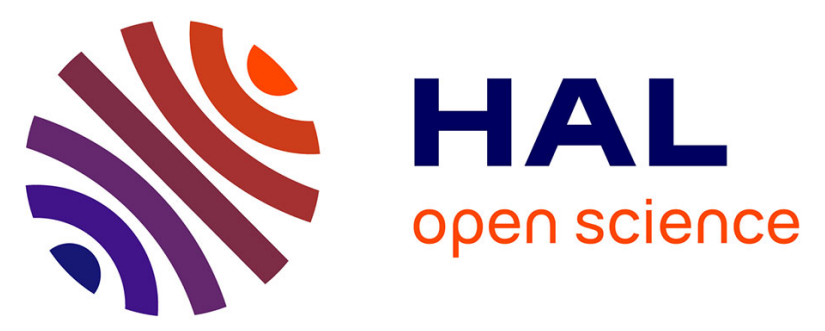

\title{
Ultrasound shear wave elastography for assessing diaphragm function within the intensive care unit
}

Thomas Poulard, Quentin Fosse, Jean-Yves Hogrel, Marie-Cecile Nierat, Thomas Similowski, Martin Dres, Damien Bachasson, Jean-Luc Gennisson

\section{- To cite this version:}

Thomas Poulard, Quentin Fosse, Jean-Yves Hogrel, Marie-Cecile Nierat, Thomas Similowski, et al.. Ultrasound shear wave elastography for assessing diaphragm function within the intensive care unit. 2019 IEEE International Ultrasonics Symposium (IUS), Oct 2019, Glasgow, United Kingdom. pp.966969, 10.1109/ULTSYM.2019.8925575 . hal-03366516

\section{HAL Id: hal-03366516 https://hal.sorbonne-universite.fr/hal-03366516}

Submitted on 5 Oct 2021

HAL is a multi-disciplinary open access archive for the deposit and dissemination of scientific research documents, whether they are published or not. The documents may come from teaching and research institutions in France or abroad, or from public or private research centers.
L'archive ouverte pluridisciplinaire HAL, est destinée au dépôt et à la diffusion de documents scientifiques de niveau recherche, publiés ou non, émanant des établissements d'enseignement et de recherche français ou étrangers, des laboratoires publics ou privés. 


\title{
Ultrasound shear wave elastography for assessing diaphragm function within the intensive care unit
}

\author{
Thomas Poulard ${ }^{1,3}$, Quentin Fosse ${ }^{2}$, Jean-Yves Hogrel ${ }^{3}$, Marie-Cécile Niérat ${ }^{2}$, Thomas Similowski ${ }^{2}$, Martin Dres ${ }^{2}$, \\ Damien Bachasson ${ }^{1 *}$, Jean-Luc Gennisson ${ }^{3 *}$ \\ ${ }^{1}$ Neuromuscular Investigation Center, Institute of Myology, PARIS (France) \\ ${ }^{2}$ Sorbonne Université, INSERM, UMRS1158 Neurophysiologie Respiratoire Expérimentale et Clinique ; AP-HP, \\ Groupe Hospitalier PitiéSalpêtrière Charles Foix, Service de Pneumologie, Médecine intensive et réanimation, \\ Département R3S, PARIS (France) \\ ${ }^{3}$ IR4M, CNRS UMR 8081, Université Paris-Saclay, CEA Service Hospitalier Frédéric Joliot, ORSAY (France)
}

\begin{abstract}
Availability of robust non-invasive tools for assessing diaphragm function is clinically relevant in a variety of clinical settings. Diaphragm shear modulus (SMdi) assessed by using ultrasound (US) shear wave elastography (SWE) can be used as a surrogate of transdiaphragmatic pressure (Pdi) that is considered as the most specific approach to assess diaphragm function. Here, we investigated the ability of SWE to reveal changes in transdiaphragmatic pressure for assessing diaphragm function within an ICU setup. SWE was performed in 22 mechanically ventilated patients within varying breathing conditions to induce changes in Pdi. Eight patients displayed a significant correlation between changes in Pdi ( $\Delta$ Pdi) and changes in SMdi ( $\Delta$ SMdi). An absence of correlation was associated with a significantly higher respiratory rate. These findings highlight the need to increase SWE sampling rate to improve the applicability of SWE for diaphragm monitoring.
\end{abstract}

Keywords-diaphragm, ultrafast ultrasound imaging, shear wave elastography, mechanical ventilation, intensive care unit, muscle

\section{INTRODUCTION}

The diaphragm being the main respiratory muscle, assessing its efficiency is of primary importance in various populations. Diaphragm dysfunction is highly prevalent in patients admitted in the intensive care unit (ICU) and is associated with prolonged ICU-stay, poorer prognosis and/or death [1]. Diaphragm function may be estimated by measuring transdiaphragmatic pressure (Pdi). Pdi measurement requires the insertion of nasogastric catheters through the nose or mouth of the patient and is computed as the difference between the gastric pressure (Pga) and the esophageal one (Pes). Since the diaphragm is the only muscle responsible for a concomitant decrease in Pes and an increase in Pga, Pdi is considered as the gold standard method to assess diaphragm force generation. In addition to invasiveness, Pdi is also an indirect measure of diaphragm contractility [2].

Ultrasound imaging of the diaphragm has been widely used to assess its function [3]. Diaphragm may easily be imaged via an intercostal approach during which diaphragm thickness and

Supported by the AFM "Association Française contre les Myopathies" jointly with "Fondation EDF". thickening fraction can be measured, or via a subcostal approach to measure its excursion. The ease of use of diaphragm ultrasound has led to a vast body of research on this topic. However, equivocal relationships between Pdi and diaphragm thickening fraction have been reported [4], supporting the need to investigate innovative techniques to improve diaphragm function assessment. Our group has recently shown in healthy subjects that inspiratory changes in diaphragm shear modulus ( $\triangle$ SMdi) measured using ultrasound shear wave elastography (SWE) are strongly correlated to changes in Pdi ( $\Delta \mathrm{Pdi},[5])$. The aim of the present study was to investigate the suitability of SWE to assess diaphragm function in mechanically ventilated patients within the ICU.

\section{MATERIAL \& METHODS}

\section{A. Patients}

All patients or their legal representative gave written informed consent. This study conformed to the Declaration of Helsinki and was approved by the local ethics committee. Patients had to be 18 and over, receiving mechanical ventilation for more than 24 hours and able to perform a spontaneous breathing trial (SBT, i.e. breathing while receiving no ventilator support). Out of the twenty-five patients, three of them were not considered for analysis (two for an incomplete set of data, one because of complete diaphragm paralysis). Patients were studied in a semirecumbent position (around 40 degrees).

\section{B. Ultrasound recording}

An ultrafast ultrasound scanner (Aixplorer, Supersonic Imagine, France) driving with a linear transducer array (SL102, Supersonic Imagine) was used to scan the right hemidiaphragm. The probe was placed on the anterior axillary line vertical to the chest wall, at the $8^{\text {th }}-11^{\text {th }}$ intercostal space. The ultrasound sequence was designed to optimize the pushing beam in the diaphragm. Briefly, ultrasounds were focused along one line to create radiation force that acted as a shear wave source. 


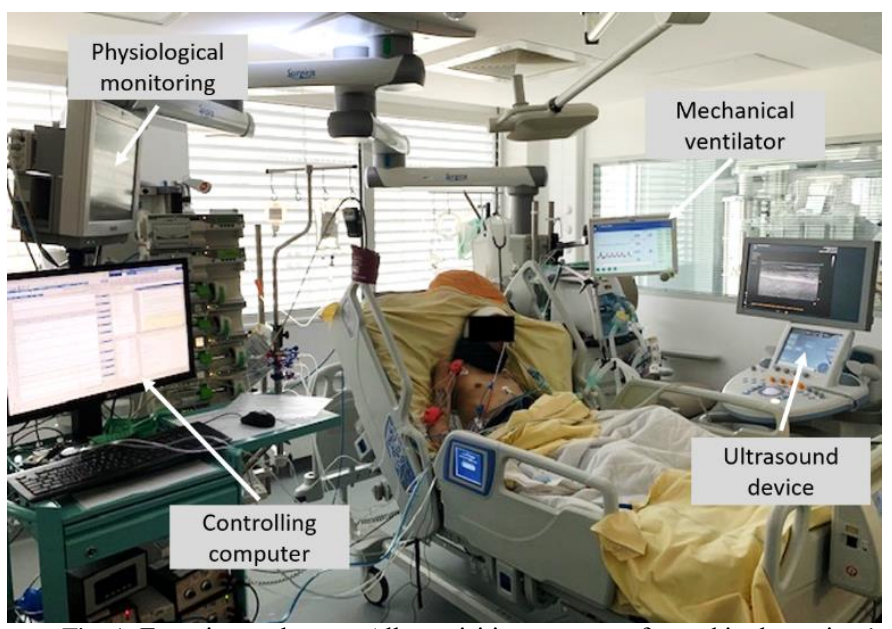

Fig. 1. Experimental setup. All acquisitions were performed in the patient's room. The ultrasound device was connected to a controlling computer, which was synchronizing and recording ultrasound images (SWE and Bmode) and physiological data.

Then the propagating shear wave was captured using an ultrafast imaging sequence (3500 frames. $\mathrm{s}^{-1}$ ) allowing its velocity measurement. By using a Matlab interface, B-Mode images and SWE maps where retrieved. The conventional SWE framerate of the device is set to $1 \mathrm{~Hz}$. The SWE sequence was then modified to reach $2 \mathrm{~Hz}$ and sometime decreased to $1.6 \mathrm{~Hz}$ depending on diaphragm depth.

\section{Physiological parameters}

Various physiological parameters were recorded simultaneously with the ultrasound acquisitions. Pga and Pes were measured using balloon catheters (NutriVent $^{\mathrm{TM}}$, Mirandola, Modena, Italy) inserted through the mouth or nose of the patient and connected to separated differential pressure transducers (model DP45-32, Validyne, Northridge, CA). Pdi was computed as the difference between Pga and Pes. Heart rate, breathing frequency, oxygen saturation and arterial blood pressure were continuously monitored using in-room monitoring systems. Flow was measured using a flow sensor (Hamilton Medical, Bonaduz, Switzerland) connected to a spirometer (ADInstruments, Bella Vista, Australia). All signals were analogue-to-digitally converted at a sampling rate of $1 \mathrm{kHz}$ using a PowerLab system (16/35, ADInstruments, Bella Vista, Australia).

All signals (i.e. ultrasound data and physiological parameters) were synchronized using a trigger output sent by the ultrasound scanner to the PowerLab when the ultrasound acquisition started. The experimental setup is presented in Fig. 1.

\section{Breathing conditions}

Variations in diaphragmatic work during breathing was induced by changing ventilator parameters. Thirty seconds acquisitions were performed at 3 and 9 minutes after the start of each condition. Afterward, a SBT was performed during which the patient was breathing without receiving help from the ventilator. Thirty seconds recordings were performed every five minutes with a maximal duration set at 30 minutes.

\section{E. Data processing and statistical analysis}

All data were analyzed offline using standardized MATLAB scripts. Regarding SWE maps, a region of interest (ROI) was defined in the diaphragm for every tested condition in which shear modulus values were averaged. A shape preserving interpolation was then applied to transform shear modulus values at $1 \mathrm{kHz}$, so that diaphragm shear modulus (SMdi) could be easily compared to Pdi. $\Delta$ SMdi was computed as the difference between maximal SMdi minus minimal SMdi over the breathing cycle. $\Delta$ Pdi was computed as the difference between maximal Pdi minus minimal Pdi over the breathing cycle

Pearson's correlation ( $\mathrm{r}$ ), repeated measure correlation ( $\mathrm{R}$ [6]) were performed to evaluate the relationship between $\Delta$ SMdi and $\Delta$ Pdi. Differences between patients who failed and patients who succeeded the SBT were assessed through Student's $t$-test. All analysis were performed in the computing environment $\mathrm{R}$ (version 3.2.4, $\mathrm{R}$ Foundation for Statistical Computing, Vienna, Austria [7]). Statistical significance was set at $\mathrm{p}<0.05$.

\section{RESULTS}

\section{A. Typical recording of diaphragm SWE}

Fig. 2. displays a typical imaging of the diaphragm using SWE. The diaphragm is classically identified by the two echogenic pleural and peritoneal membranes bounding the muscular layer.

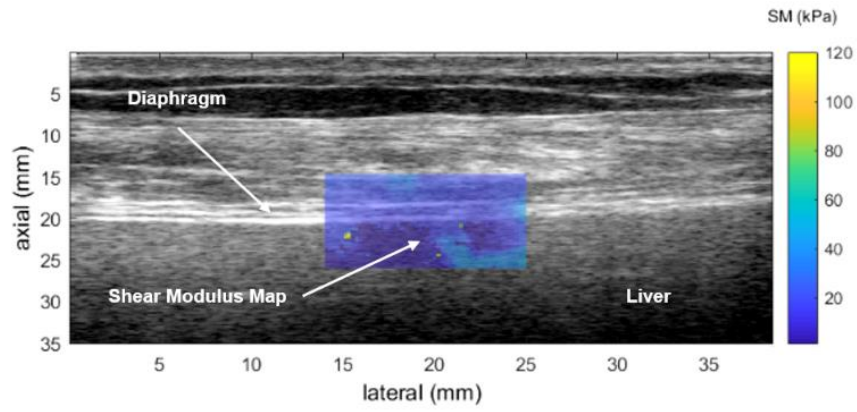

Fig. 2. Shear modulus (SMdi) map in $\mathrm{kPa}$ measured using shear wave elastography overlaid with standard B-Mode during intercostal scanning at the right zone of apposition.

\section{B. Relationship between $\triangle S M d i$ and $\triangle P d i$}

Relationship between $\triangle \mathrm{SMdi}$ and $\Delta \mathrm{Pdi}$ are presented for two patients. One of them displayed a significant correlation $(\mathrm{r}=$ $0.88, \mathrm{p}<0.001)$, while the other showed no correlation $(\mathrm{r}=-$ $0.05, \mathrm{p}=0.88)$.

The patient that displayed significant $\Delta$ SMdi- $\Delta$ Pdi correlation had a significantly lower respiratory rate compared to the patient with no significant $\Delta \mathrm{SMdi}-\Delta$ Pdi correlation $(\mathrm{p}<0.02)$. 


\section{DISCUSSION AND PERSPECTIVES}

This study investigated whether SWE could be reliably used to evaluate diaphragm contractility in critically ill patients. These preliminary findings are promising although the use of SWE for diaphragm assessment may not be generalized to all patients.
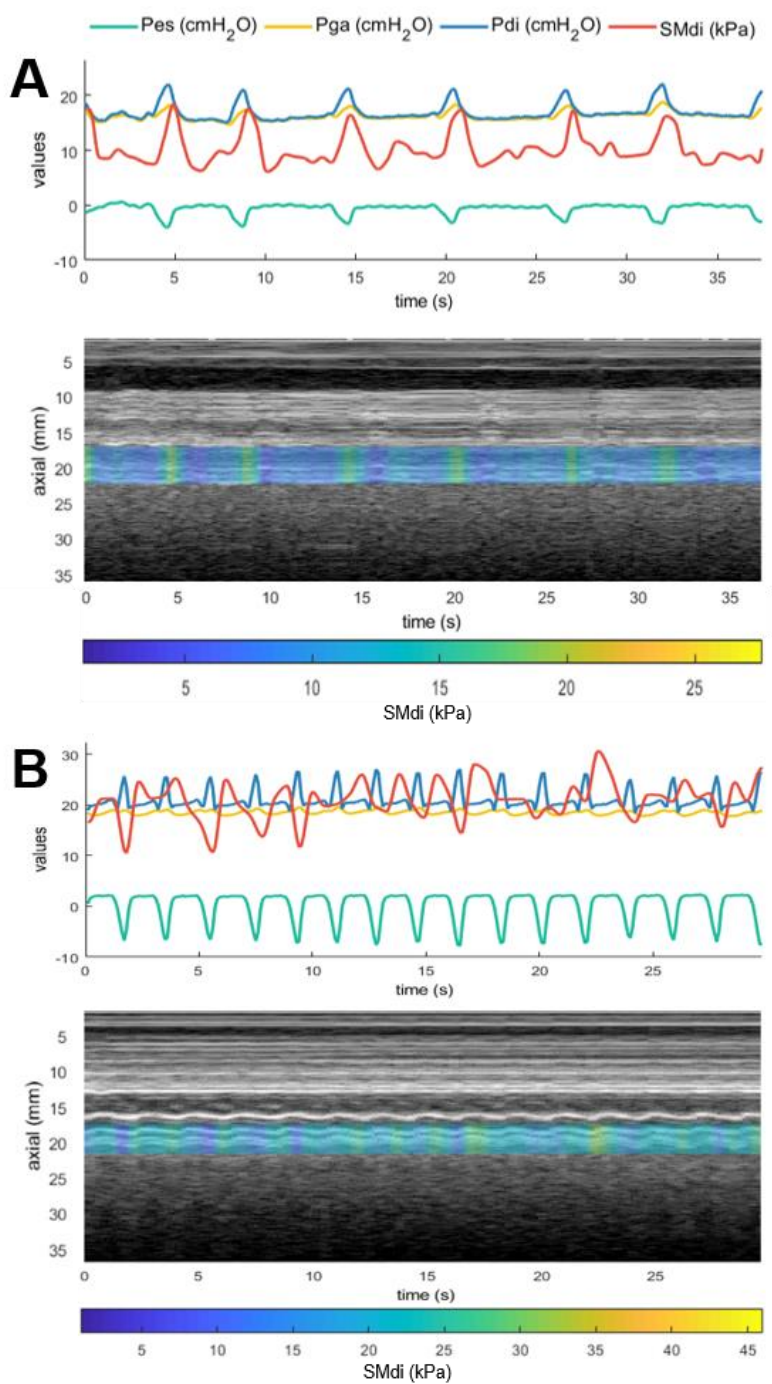

Fig. 3. Variations of diaphragm shear modulus (SMdi, red), esophageal (Pes, green), gastric (Pga, yellow) and transdiaphragmatic (Pdi, blue) pressures over time, for a patient with a very strong correlation between $\triangle$ Pdi and $\Delta$ SMdi $(\mathrm{A}, \mathrm{r}$ $=0.88)$ and a patient with a null correlation $(\mathrm{B}, \mathrm{r}=-0.05)$. Time-Motion imaging overlaid with shear modulus maps are also displayed.

The fact that absence of $\Delta \mathrm{SMdi}-\Delta \mathrm{Pdi}$ correlation was associated with a higher respiratory rate suggest that low SWE sampling rate hampers the ability of SWE to monitor diaphragm activity. As stated above, shear modulus values were acquired at 1.6-2 Hz. Therefore, an increased respiratory rate results in a decreased number of shear modulus values per breathing cycle while the probability to oversee within-cycle maximal and minimal shear modulus increases. Consequently, the risk to underestimate $\Delta \mathrm{SMdi}$ is growing with respiratory rate. Fig. 3 shows typical recording of physiological parameters and SMdi over time in two patients with strong (Fig. 3A) and absent (Fig. 3B) $\Delta$ SMdi- $\Delta$ Pdi correlation. One may clearly observe that an increased respiratory rate (Fig. 3B) negatively impacts SWE ability to monitor changes in SMdi. This supports that the low success rate of diaphragm SWE in patients is mainly related to technological limitations.

Conventional SWE sequences used in the present work involves four ultrasound pushing beams generated over multiple laterally-spaced spatial points for generating shear waves. To keep the sequence within the FDA guidance and to increase the framerate, a new sequence reducing the number of pushing lines to two was designed in order to obtain $4-\mathrm{Hz}$ framerate. Then a multiple tracking location (MTL) algorithm allows the computation of shear wave velocity maps. A recent work reported that a two-point measurement of shear wave velocity can be reliably used to assess the mechanical properties of viscoelastic material [8]. This technique is very promising as a single (i.e. instead of four) ultrasound pushing beam would be sufficient to map a predefined area. This would lead to a four times higher sampling frequency than what is actually used while remaining within the limits of radiation exposure. However, its applicability to the skeletal muscle remains to be investigated.

These findings support that SWE is a promising noninvasive alternative for monitoring diaphragm function. However, the generalization of SWE for the diaphragm monitoring depends upon technological developments to overcome current limitation of commercially available SWE sequences.

\section{REFERENCES}

[1] Dres, M., Goligher, E. C., Heunks, L. M., \& Brochard, L. J. (2017). Critical illness-associated diaphragm weakness. Intensive Care Medicine, 43(10), 1441-1452.

[2] S. American Thoracic Society/European Respiratory, "ATS/ERS Statement on respiratory muscle testing", am J Respir Crit Care Med, vol. 166, no. 4, pp. 518-624, Aug 15, 2002

[3] Nason, L. K., Walker, C. M., McNeeley, M. F., Burivong, W., Fligner, C. L., \& Godwin, J. D. (2012). Imaging of the diaphragm: anatomy and function. Radiographics, 32(2), E51E70.

[4] Goligher, E. C., Laghi, F., Detsky, M. E., Farias, P., Murray, A., Brace, D., ... \& Ferguson, N. D. (2015). Measuring diaphragm thickness with ultrasound in mechanically ventilated patients: feasibility, reproducibility and validity. Intensive care medicine, 41(4), 642-649.

[5] Bachasson, D., Dres, M., Niérat, M. C., Gennisson, J. L., Hogrel, J. Y., Doorduin, J., \& Similowski, T. (2019). Diaphragm shear modulus reflects transdiaphragmatic pressure during isovolumetric inspiratory efforts and ventilation against 
inspiratory loading. Journal of Applied Physiology, 126(3), 699-707.

[6] Bakdash, J. Z., \& Marusich, L. R. (2017). Repeated measures correlation. Frontiers in psychology, 8, 456.

[7] Team, R. C. (2014). A language and environment for statistical computing. Vienna, Austria: R Foundation for Statistical Computing.
[8] Kijanka, P., Ambrozinski, L., \& Urban, M. W. (2019). Two Point Method For Robust Shear Wave Phase Velocity Dispersion Estimation of Viscoelastic Materials. Ultrasound in medicine \& biology. 